\title{
Identifying Effective Safety Planning Tasks for
}

\section{Construction Management}

\author{
Kang, Sang Hun, Kim, Dae Young \\ Pusan National University / Department of Architectural Engineering
}

\begin{abstract}
The main purpose of the study is to reduce the accident death rate in the construction industry. To achieve this goal, the safety planning tasks were analyzed and assessed in terms of the importance and preventive effects of the tasks. Through the extensive literature reviews, the safety planning tasks were identified and reviewed by safety managers. Based on the data collected, a survey was conducted in order to receive responses by experts with knowledge on the effects and importance of preventive measures for safety. Then, the IPA method was employed to identify the safety manager's tasks that have an effect on performance indicators. The effective safety tasks identified are expected to have a critical role in reducing the accident death rate in the construction industry.
\end{abstract}

Keywords: importance; performance; preventive; safety manager; survey; 